\title{
Aproximaciones al Principio de Autonomía en el trata- miento de diálisis: el Consentimiento Informado y las Voluntades Anticipadas
}

\author{
Maribel Díaz Jurado - Nieves Simal Vélez - Esther Salillas Adot - Maricel Julve Ibáñez
}

Servicio de Nefrología del Hospital Universitario de Bellvitge. Barcelona

\section{Resumen}

En la actualidad el Principio de Autonomía pretende marcar la relación asistencial entre los profesionales sanitarios y los pacientes. Actualmente, la expresión del Principio de Autonomía del paciente en las decisiones referidas a su tratamiento se plasma en el Consentimiento Informado y el Documento de Voluntades Anticipadas. El objetivo de este trabajo, es verificar que conocimientos tiene el paciente con insuficiencia renal crónica terminal en tratamiento de diálisis en nuestro hospital, referido al Consentimiento Informado y el Documento de Voluntades Anticipadas y saber si el paciente se consideró bien informado sobre su estado de salud al inicio de su enfermedad renal y en la actualidad.

Estudio retrospectivo, observacional y descriptivo en 55 pacientes de nuestro servicio. Los resultados muestran que el Consentimiento Informado no es conocido por la mayoría de los pacientes y que el Documento de Voluntades Anticipadas no forma parte de la información que recibe. La información recibida al inicio del tratamiento es satisfactoria en todos los pacientes. En la actualidad están más satisfechos los pacientes en diálisis peritoneal y también han tenido más posibilidad de elegir tratamiento. Los enfermos en hemodiálisis desean tener más autonomía en las decisiones referidas a su tratamiento.

\section{Correspondencia: \\ Maribel Díaz Jurado \\ Servicio de Nefrología \\ Hospital Universitario de Bellvitge \\ Feixa Llarga s/n - 08907 L'Hospitalet. Barcelona \\ midiaz@bellvitgehospital.cat}

PALABRAS CLAVE:

PRINCIPIO DE AUTONOMÍA

- CONSENTIMIENTO INFORMADO

- DOCUMENTO DE VOLUNTADES ANTICIPADAS

- INSUFICIENCIA RENAL

- DIÁLISIS

Approaches to the Principle of Autonomy in dialysis treatment: Informed Consent and Living Will

\section{Abstract}

At present, the principle of autonomy seeks to provide the framework for relations between healthcare professionals and patients in the context of their care. Currently, the expression of the principle of the patient's autonomy in decisions concerning his or her treatment is materialized in the Informed Consent and Living Will. The aim of this work is to verify what knowledge terminal chronic renal insufficiency patients undergoing dialysis in our hospital have, with regard to Informed Consent and the Living Will and to know whether patients consider themselves to be well-informed about the state of their health at the start of their kidney disease and at present.

A retrospective, observational and descriptive study of 55 patients of our service. The results show that Informed Consent is unknown to the majority of the patients and that the Living Will is not part of the information they receive. The information received at the start of treatment is satisfactory in the case of all the patients. At present, patients undergoing peritoneal dialysis are more satisfied 
and have also had the possibility of choosing their treatment. Patients on haemodialysis would like greater autonomy in the decisions concerning their treatment.

\section{KEY WORDS:}

PRINCIPLE OF AUTONOMY

INFORMED CONSENT

LIVING WILL

RENAL INSUFFICIENCY

DIALYSIS

\section{Introducción}

El Principio de Autonomía, junto con los Principios de Beneficencia, No Maleficencia y Justicia han ser los principios éticos en los que en la actualidad debe basarse toda relación asistencial. Estos Principios son los que deben utilizarse para discutir cuestiones bioéticas fundamentales. La búsqueda del concepto de Autonomía de las personas ha sido una inquietud presente desde hace muchos años. Dos filósofos muy relevantes del siglo XVIII: Inmanuelle Kant y J.S Mill hablaban de la Autonomía como de un derecho inherente al ser humano.

Kant la definía como "la capacidad que tiene cada individuo de darse a sí mismo normas que dicten su conducta. También J.S. Mill hablaba de la Autonomía como autogobierno: "Es decidir por uno mismo al margen de leyes y normas externas. La única restricción es no hacer daño a los demás".

Es en la "Declaración de los Derechos de los Pacientes", publicada en EE UU en el año 1973, donde se afirma que el paciente en nombre de su autonomía tiene derecho a la información sobre su salud y al consentimiento informado. El Informe Belmont, creado en EE UU en el año 1978, habla por primera vez del respeto a la Autonomía de las personas en lo que se refiere a la experimentación con seres humanos, mediante un consentimiento informado. En este informe se destacan tres elementos imprescindibles para que el paciente pueda tomar decisiones. Estos son: Información, Comprensión y Voluntariedad. Un año después, en 1979, Beauchamp y Childress en su libro "Principios de ética Biomédica" hablan de la aplicación del Principio de Autonomía en el ámbito asistencial, así como del resto de Principios Éticos Básicos. En 1997, el Consejo de Europa a través del "Convenio relativo de los Derechos Humanos y la Biomedicina" ó Convenio de Oviedo, habla de que una intervención en el ámbito de la sanidad solo podrá efectuarse después de que la persona afectada haya dado su consentimiento de forma libre e inequívoca. En el año 2002 la ley 41/2002 regula la autonomía del paciente y de sus derechos y obligaciones en materia de información y documentación clínica.

El Principio de Autonomía hace referencia a la posibilidad del enfermo, si así lo desea, de tomar libremente decisiones referidas a su salud y posibles tratamientos. En los últimos veinte años se ha producido un cambio en la relación sanitaria entre los profesionales sanitarios y el paciente. La relación actual ya no se basa en actitudes "paternalistas" donde se hacía lo mejor para el enfermo, pero solo desde la perspectiva del médico, sin tener en cuenta la opinión del enfermo ó incluso contra su voluntad.

El Principio de Autonomía en la actualidad pretende marcar la relación asistencial entre los profesionales sanitarios y los pacientes. Respetar la autonomía del paciente requiere de los profesionales una información clara, verídica y adecuada a la capacidad de comprensión del paciente sobre su enfermedad, su evolución y el tratamiento que van a realizarle. El enfermo ha de argumentar su decisión de forma lógica y continua en el tiempo. Ha de haber proporcionalidad entre su decisión y las consecuencias que de ella se derivan. Hay situaciones en que el paciente confunde autonomía con obstinación y preferentismo. El cree que la decisión tomada es correcta, pero no tiene capacidad de diálogo ni argumentos sólidos para justificarla. Se ha de tener en cuenta que, en muchas ocasiones, las condiciones en que el paciente recibe toda la información sobre su nueva situación están marcadas por la vulnerabilidad en que se encuentra. El tiempo para decidir es limitado y el miedo a la enfermedad influye en la toma de decisiones.

La expresión del Principio de Autonomía de los pacientes en las decisiones referidas a su tratamiento se plasma en:

- El Consentimiento Informado (CI): Es un proceso verbal por el que el paciente, una vez ha 
sido convenientemente informado por el médico responsable, accede a realizarse la intervención que éste le propone. Puede acompañarse de un documento que sirve de garantía de que el paciente ha recibido la información pertinente. Este documento nunca ha de sustituir la información verbal.

- El Documento de Voluntades Anticipadas (DVA) ó Testamento Vital: Documento en el que la persona titular expone de forma anticipada las instrucciones sobre las actuaciones médicas que se deberán tener en cuenta en una situación en la que las circunstancias no le permitan expresar libremente su voluntad.

Para prestar el CI y las VA el enfermo ha de ser competente, recibir una información adecuada, comprenderla y actuar voluntariamente. En el caso del DVA, se pretende respetar las decisiones aun cuando éste no pueda hacerlo debido a una situación de máxima vulnerabilidad.

En la actualidad, en Cataluña, existe la obligatoriedad de cumplimentar el CI (Ley 21/2000) y adjuntarlo a su Historia Clínica. También el Parlamento Español emite la Ley 41/2002 que regula la Autonomía de los pacientes y recoge la obligatoriedad del CI.

La posibilidad de expresar anticipadamente los deseos del paciente en el DVA, no ha sido recogida en el ordenamiento jurídico estatal. A partir de la ratificación del Convenio de Oviedo, en 1997, se ha abierto la puerta para su regulación por parte de las Comunidades Autónomas. En nuestro hospital en los últimos tiempos, desde la Consulta Externa, se está intentando hacer más partícipe al enfermo con IRC en la información referente a su salud, sus cuidados y las posibles opciones de Tratamiento Renal Sustitutivo (TRS).

\section{Ojetivo Principal}

Verificar que conocimientos tiene el paciente con Insuficiencia Renal Crónica Terminal en tratamiento de diálisis (hemodiálisis/diálisis peritoneal) en nuestro hospital, referidos al Consentimiento Informado y a las Voluntades Anticipadas.

\section{Ojetivos Secundarios}

1. Saber si el paciente se consideró bien informado sobre su estado de salud al inicio de su enfermedad renal y en la actualidad.

2. Conocer que información recibió el paciente con insuficiencia renal terminal de las dos opciones de diálisis antes de iniciar el Tratamiento Renal Sustitutivo y posibilidad de elección.

3. Conocer si el paciente desea tener más Autonomía en las decisiones referidas a su tratamiento.

4. Analizar que aportaciones hace Enfermería en la consecución del Consentimiento Informado.

\section{Material y Método}

Se procedió a una revisión bibliográfica referida al CI y a las VA, así mismo se revisaron los impresos que hay en nuestro hospital referidos al CI, las VA y la información sobre el tratamiento de diálisis.

En una segunda fase, se elaboró una encuesta $a d$ hoc que consta de 12 preguntas cerradas referidas al $\mathrm{CI}$, las VA, y la información recibida por el paciente al inicio de su enfermedad renal y en la actualidad. Tras informar a los pacientes entrevistados de los objetivos del estudio y solicitar su consentimiento verbal, se entregaron un total de 55 encuestas. El diseño del estudio es retrospectivo, observacional y descriptivo.

La muestra estudiada han sido los pacientes en programa de diálisis (HD/DP) de nuestro hospital. De los 36 pacientes en programa de hemodiálisis 5 no entraron en el estudio por no ser legalmente competentes y depender de un tutor legal que no fue entrevistado, y uno cumplimentó mal la encuesta. De Diálisis Peritoneal fueron entrevistados al azar 25 de los 40 pacientes en programa.

Para el análisis de datos se ha utilizado el programa estadístico Stat View. 


\section{Resultados}

\section{Perfil del paciente}

La edad media de los pacientes estudiados es $61 \pm 15$ años, de los cuales un $64 \%$ eran hombres y un $36 \%$ eran mujeres. El 56\% tenían estudios primarios y el $7 \%$ eran universitarios.

\section{Respecto al CI}

Los pacientes tratados con DP saben que es el CI un $48 \%$, frente a un $52 \%$ que dice no conocerlo. Los pacientes en HD saben que es el CI un $36 \%$ y un $60 \%$ responde que no lo sabe.

Según las respuestas, han firmado el CI el $16 \%$ de los pacientes en DP y el $40 \%$ de los pacientes en HD. Hay 7 pacientes que dicen no conocer el CI, y sin embargo responden que si lo han firmado.

\section{Voluntades Anticipadas}

De los 55 pacientes que han sido encuestados, 10 pacientes responden afirmativamente que conocen el DVA, 6 en programa de HD y 4 en programa de DP. La prensa ha sido la fuente de información en la mayoría de los casos. Solo 3 personas han sido informadas por el equipo asistencial.

Información Inicial recibida por el paciente al inicio de su enfermedad renal y en la actualidad

Todos los pacientes en DP se consideran bien informados al inicio de su enfermedad renal y también en la actualidad (25.9 \pm 24 meses después del inicio). Referente a HD se consideran bien informados al inicio el $90 \%$ y en la actualidad el $70 \%(41 \pm 28$ meses después del inicio), $(p=0,02)$.

\section{Información de las dos opciones de tratamiento (HD/DP) y posibilidad de elección}

El $88 \%$ de los pacientes en DP fueron informados de las dos opciones de tratamiento, antes de iniciarlo, frente a un $50 \%$ de los pacientes en HD que no conocían las dos opciones de tratamiento antes de comenzar ( $p=0,08$ ), (figura 1 ). La posibilidad de elección de tratamiento por parte de los pacientes es del $84 \%$ en caso de DP y de un $23 \%$ en HD ( $p=0,0004)$, (figura 2).

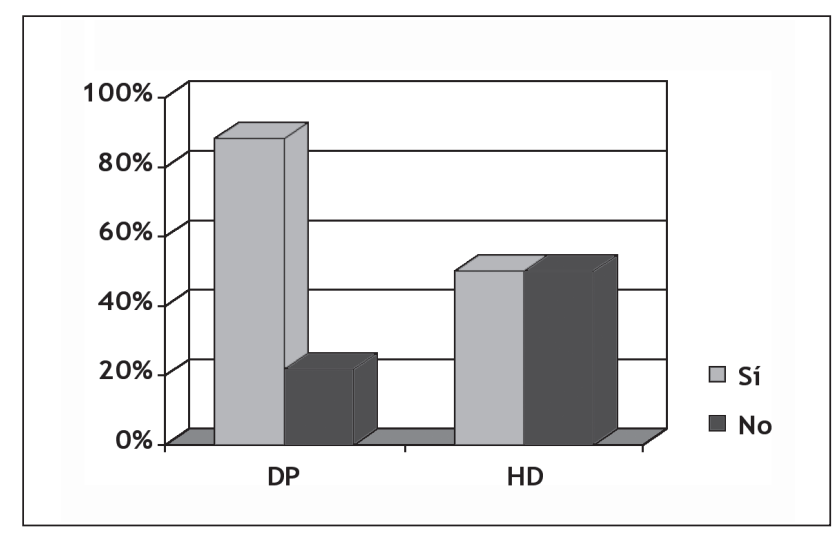

Figura 1. Conocimiento de las dos opciones de trataiento

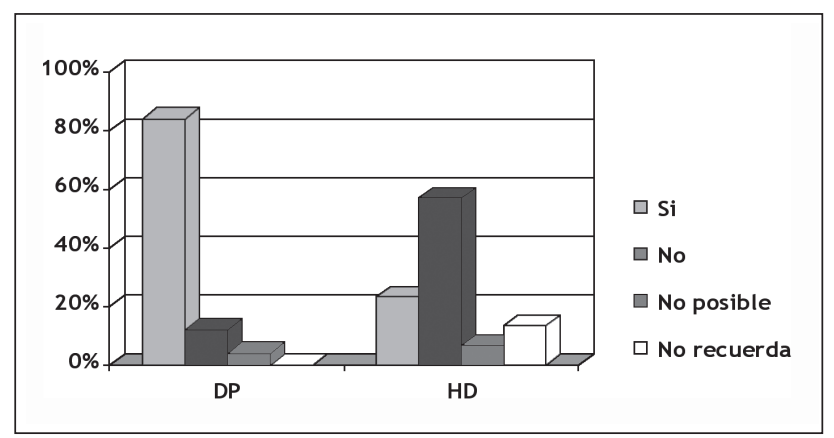

Figura 2. Posibilidad de elección de tratamiento

Autonomía en las decisiones referidas a su tratamiento

Respecto a si el paciente desea tener más Autonomía en las decisiones referidas a su tratamiento, los pacientes en DP responden negativamente un $64 \%$ frente a un $66 \%$ de enfermos en HD que si responden afirmativamente. $(p=0,002)$.

\section{Discusión}

Observamos el bajo conocimiento del CI por parte de los pacientes estudiados, sólo lo conoce un $42 \%$, lo cual indica que en muchos casos se ha reducido a la firma de un papel, sin entender lo que quiere significar. EI CI no es la firma de un documento cuyo valor solo es legal. Ha de ser el resultado de una relación asistencial entre el enfermo y el personal sanitario, donde la información dada al paciente y su comprensión, así como la autonomía en las decisiones tomadas por éste, sean prioritarias. EI DVA la sociedad no lo percibe como algo propio, y por tanto los pacientes en tratamiento de diálisis tampoco lo conocen. La posibilidad de incluir este documento en la Historia 
Clínica de paciente no es ofrecida en la información dada por el equipo asistencial. En Cataluña hay registrados en el Departamento de Salud 8000 DVA lo que representa un $0,1 \%$ de la población residente en Cataluña.

La información inicial dada al enfermo próximo a iniciar Tratamiento Renal Sustitutivo ha de ser clara y lo más adecuada posible a cada paciente. Debe informarse de las diferentes opciones de tratamiento, si no hay contraindicación médica de ninguna de ellas para el enfermo. Es posible que la detección precoz de la IRC influya en poder elegir con tiempo la técnica. Los pacientes en programa de DP han tenido opción a la elección del tratamiento un $84 \%$ de ellos a diferencia de los que realizan HD que han podido elegir el $23 \%$.

Los enfermos tratados con DP están satisfechos de la información recibida al comienzo de su enfermedad renal y ésta satisfacción se mantiene en el tiempo $(100 \%)$. No reclaman más autonomía el $64 \%$ de ellos. Puede ser debido a que participan más activamente en todo su proceso asistencial. Los pacientes en programa de HD están satisfechos el $90 \%$ con la información recibida al inicio de su tratamiento, mientras que en la actualidad el grado de satisfacción es del $70 \%$. Reclaman más autonomía en las decisiones referidas a su tratamiento un $66 \%$. Con el tiempo, la relación de estos enfermos en HD con el equipo asistencial que lo atiende, se va haciendo cada vez más rutinaria y dependiente pudiendo verse afectada la comunicación entre ambos.

Enfermería, como parte del equipo asistencial, ha de ofrecer una información de calidad al enfermo renal. La proximidad de la enfermera con el paciente debe ayudarle a identificar carencias y dudas que éstos pueden presentar en el momento de tomar decisiones referidas a su salud y su tratamiento.

\section{Conclusiones}

- EI CI no es conocido por los pacientes en tratamiento de diálisis. Ha quedado reducido a un documento cuyo valor sólo es legal.

- Las VA no forman parte de la información que recibe el paciente en programa de diálisis.
- El grado de satisfacción de la información recibida al inicio del tratamiento es satisfactoria en los todos los pacientes (HD/ DP).

\section{Bibliografía}

1. Beauchamp T. Principios de Ética Médica. BarceIona: Editorial Masson; 2002.

2. Busquets $E$, Mir J. Infermeria i Informació i Consentiment Informat. Barcelona: Editorial IBB; 2006.

3. Camps V. La Voluntad de Vivir. Barcelona: Editorial Ariel; 2005.

4. Gracia D. Como arqueros al blanco. Madrid: Editorial Triacastela; 2004.

5. Gracia D. Procedimientos de Decisión en Ética Clínica. Madrid: Editorial Eudema; 1991.

6. Hernando L. La selección del paciente para el tratamiento sustitutivo de la IRC, ¿es posible? Rev. Nefrología 1994; Vol. XIV Suplementol.

7. Ibarzabal X. Bioética: tomando decisiones para el final de la vida. Pensando en el principio de autonomía. Rev Mult Gerontol 2004; 14(3):158-169.

8. Martínez K. Reflexiones sobre el testamento vital. Rev Atención Primaria 2003; 31(1):52-4.

9. Morey-Molina, A. Ética y nefrología. Consensos de la Sociedad Española de Diálisis y Trasplante. Rev diálisis y trasplante 2006; 27(3):102-107.

10. Portela L. Influencia del nivel de conocimiento de los pacientes sobre la elección de las distintas opciones de tratamiento. Efecto de un protocolo de información reglada, En: Libro de Comunicaciones presentadas al XXV Congreso de la SEDEN. Barcelona: HOSPAL; 2001. p. 121-127.

11. Simón P, Barrio IM. ¿Quien decidirá por mi?. Madrid: Editorial Triacastela; 2004.

12. Torralba F. Filosofía de la Medicina. Institut Borja de Bioética. 2001.

13. Wilkinson H. Información prediálisis, ¿es efectiva?. EDTNA/ EDTA Journal (ed. Española) 1998; XXIV(1): 38-40. 